\title{
Spotlight on fundus autofluorescence
}

This article was published in the following Dove Press journal:

Clinical Optometry

\author{
Ana M Calvo-Maroto' \\ Alejandro Cerviño ${ }^{2}$ \\ 'Department of Ophthalmology, \\ Clínica Universidad de Navarra, \\ University of Navarra, Madrid, \\ Spain; ${ }^{2}$ Optometry Research Group, \\ Department of Optics and Optometry \\ and Vision Sciences, University of \\ Valencia, Valencia, Spain
}

\begin{abstract}
Fundus autofluorescence (FAF) imaging is based on the fluorescence from ocular endogenous fluorophores located in the retinal pigment epithelium and choroid, mainly lipofuscin and melanin. It is a noninvasive technique that provides information about the spatial distribution of lipofuscin/melanin and retinal pigment epithelium health status. An overview about the fluorophores responsible for FAF imaging and the usefulness of FAF imaging in the noninvasive assessment and monitoring of retinal diseases, such as age-related macular degeneration, diabetic retinopathy, diabetic macular edema, central serous chorioretinopthy, retinitis pigmentosa and retinal dystrophies, are discussed in this review.
\end{abstract}

Keywords: fundus autofluorescence, short-wavelength fundus autofluorescence, near-infrared fundus autofluorescence, lipofuscin, melanin

\section{Introduction}

Autofluorescence (AF) is a phenomenon based on the capacity of certain molecules for emitting light when they are excited by suitable wavelengths. These molecules, known as fluorophores, can be divided into exogenous and endogenous. The first one is intentionally delivered into the eye for diagnosis purposes, such as in fluorescein angiography, while the presence of the second type of fluorophores is inherent in the human anatomy. Within the eye, these endogenous fluorophores are present in the cornea, crystalline lens, retinal pigment epithelium (RPE), uveal melanocytes and scleral collagen. ${ }^{1,2}$

This review aims to provide an overview focused on the AF obtained at the level of the RPE, fundus autofluorescence (FAF) and its application as a complementary tool for monitoring and assessment of retinal disease.

\section{Principles of FAF imaging}

The first observation in vivo of FAF was described by Delori et al who demonstrated that FAF at short wavelengths (SW-FAF) is emitted across a broadband from 500 to $800 \mathrm{~nm}$, and it mainly originated from lipofuscin in RPE. ${ }^{3}$ Lipofuscin is the dominant fluorophore within the retina and is a product of the oxidative breakdown of photoreceptor outer segment located in the RPE. ${ }^{4}$ The main fluorophore of RPE lipofuscin is $\mathrm{A} 2 \mathrm{E},{ }^{5}$ whose biosynthesis begins when all-trans retinal leaves the visual cycle to react with phosphatidylethanolamine (PE), resulting in the formation of A2-PE (Figure 1). Precursors of lipofuscin, mainly A2-PE and dihydroA2-PE, emit AF on the photoreceptor outer segment before phagocytosis from RPE. ${ }^{6}$ Accumulation of all-trans retinal
Correspondence: Alejandro Cerviño and Vision Sciences, University of Valencia, Dr Moliner 50, 46100 Burjassot, Valencia, Spain $\mathrm{Tel}+34963544852$

Email alejandro.cervino@uv.es 


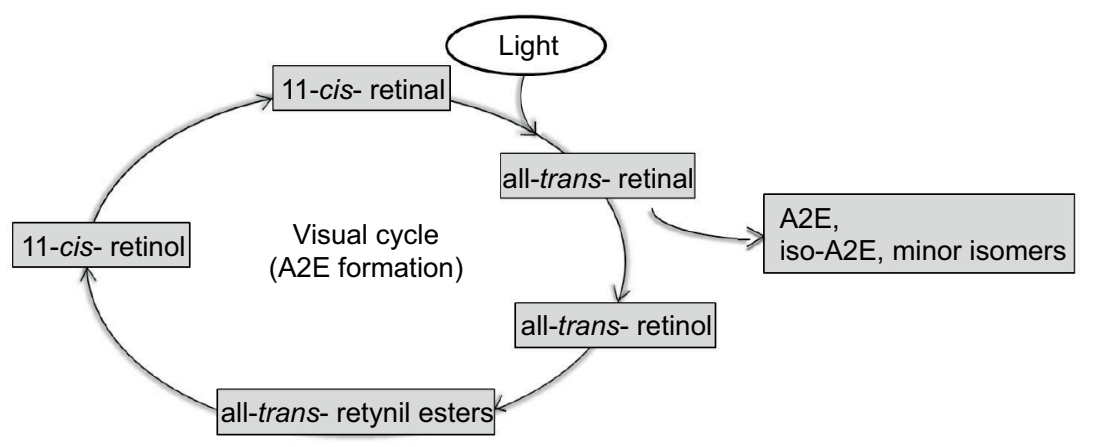

Figure I The visual cycle that represents various steps of lipofuscin metabolism and A2E formation.

in the photoreceptors leads to bisretinoid formation, which, after oxidization process, generates lipofuscin. ${ }^{6,7}$ Other lipofuscin precursors known are iso-A2E and other minor cis isomers of $\mathrm{A} 2 \mathrm{E},{ }^{6}$ and a contribution of malondialdehyde, 4-hydroxynoneual and advanced glycation end products (AGEs) to the composition of lipofuscin has also been observed. ${ }^{8}$

Thus, lipofuscin is a heterogeneous group of bisretinoids with an excitation spectrum ranging from 300 to $600 \mathrm{~nm}$ and an emission spectrum ranging from 480 to $800 \mathrm{~nm} .^{5}$

In clinical practice, FAF in vivo imaging can be obtained by commercially available devices such as confocal scanning laser ophthalmoscopy (cSLO) and digital non-mydriatic fundus cameras. The first one uses blue light excitation (488 $\mathrm{nm}$ ) and a barrier filter around $500 \mathrm{~nm}$, but it depends on the device used (Spectralis ${ }^{\circledR}$ HRA [Heidelberg]: barrier filter of 500 nm; F-10 [Nidek, Aichi, Japan]: 510 nm; Optomap ${ }^{\circledR}$ Panoramix 200 Tx [Optomap; Optos, Dunfermine, Scotland]: $540-800 \mathrm{~nm}$ ) to provide FAF imaging. However, digital non-mydriatic fundus camera uses an excitation filter around 500-580 nm and a barrier filter of 640-715 $\mathrm{nm}$ depending on the device (CX-1 and CR-2 Plus fundus cameras [Canon, Tokyo, Japan], excitation wavelengths 530-580 nm, a detection filter at $640 \mathrm{~nm}$; Visucam 200, Visucam 500, Visucam NM/FA and FF 450 Plus IR cameras [Carl Zeiss Meditec, Jena, Germany] excitation filter $510-580 \mathrm{~nm}$, a barrier filter from 650 to $735 \mathrm{~nm}$; TRC-50DX, TRC-50IX cameras [Topcon Medical Systems, Oakland, NJ, USA], excitation filter 500-610 nm, a barrier filter of 675-715 nm). ${ }^{9}$

Lipofuscin shows a yellow-orange AF when excited with ultraviolet or blue light. The signal intensity represented in SW-FAF imaging corresponds to lipofuscin accumulation that increases with aging. ${ }^{2}$

In several retinal diseases, an increase in SW-FAF intensity has been observed, which has been correlated with accumulation of lipofuscin in the RPE, due to photoreceptor outer segment phagocytosis. Moreover, it has been observed that lipofuscin is also composed of peroxidation products of proteins and lipids. Thus, lipofuscin seems to be an indicator of oxidative damage. ${ }^{10}$

Another fluorophore observed is melanin, which is located within the RPE and choroides. Melanin AF is obtained by near-infrared FAF (NIR-FAF) imaging that provides information on its distribution in the RPE cell/choroid complex. This imaging technique was first investigated by Keilhauer and Delori who used a cSLO with an excitation wavelength of $787 \mathrm{~nm}$ and a barrier filter of around $800 \mathrm{~nm} .{ }^{11}$

With aging, the number of melanosomes in RPE decreases, and an increase of melanolipofuscin and melanolysosomes occurs. ${ }^{12}$ The isolated melanin shows excitation maximum shifts from 350 to $450 \mathrm{~nm}$ and emission maximum from 440 to $560 \mathrm{~nm}$. Like lipofuscin, the intensity of melanin AF increases with aging. Furthermore, melanolipofuscin granules show fluorescence with an excitation maximum at $364 \mathrm{~nm}$ and an emission maximum at $540 \mathrm{~nm}^{2}$

Other fluorophores, such as proteins (hemoglobin and mitochondrial enzymes), flavin, flavoproteins and macular pigments, are also present on the retina. ${ }^{1}$

\section{Characteristics of normal FAF imaging}

FAF imaging allows the topographic representation of lipofuscin/melanin distribution in the RPE, which corresponds to the intensity of the signal emitted. In FAF imaging technique, dark pixel values represent low emission intensities, and bright pixel values correspond to high emission intensities.

In healthy eyes, FAF signal intensity shows a characteristic distribution where the optic nerve head appears dark due to absence of RPE and no lipofuscin, and retinal vessels show a reduced FAF signal due to absorption by blood. However, FAF pattern from the macular area depends on the type of FAF technique used; when the SW-FAF technique is employed for 
acquiring FAF imaging, the macular area, especially around the fovea AF signal, is reduced due to the absorption of macular pigments such as lutein, zeaxanthin and other pigments. ${ }^{7}$ The AF signal of parafoveal area tends to be higher than that of the fovea; however, when it is compared with the background signal in the peripheral area of the retina, it shows a reduced intensity ${ }^{13}$ (Figure 2). In NIR-FAF imaging, fluorescence signal in the central macula is high (hyperautofluorescence) due to increased optical density of melanin in an area of $\sim 8^{\circ} .{ }^{11}$ Outside of the central macula, there is an increase of melanin and a decrease in the concentration of lipofuscin in the central RPE cells, ${ }^{13}$ which corresponds to high intensity when NIR-FAF technique is used. ${ }^{11}$

\section{Clinical applications of FAF imaging}

This section shows the usefulness of FAF imaging for assessment and monitoring of several retinal diseases such as agerelated macular degeneration (AMD), diabetic retinopathy (DR), central serous chorioretinopathy (CSC), retinitis pigmentosa (RP) and macular dystrophies.

Previously, we have explained lipofuscin/melanin distribution in normal eyes; therefore, any change in this FAF pattern can be easily identified, and its qualitative assessment is widely used for monitoring certain retinal diseases.

FAF intensity abnormalities are classified into hypoautofluorescent signal, wherein FAF is abnormally dark due to a reduced lipofuscin concentration in the RPE, an increase in melanin concentration or even signal absorption from the

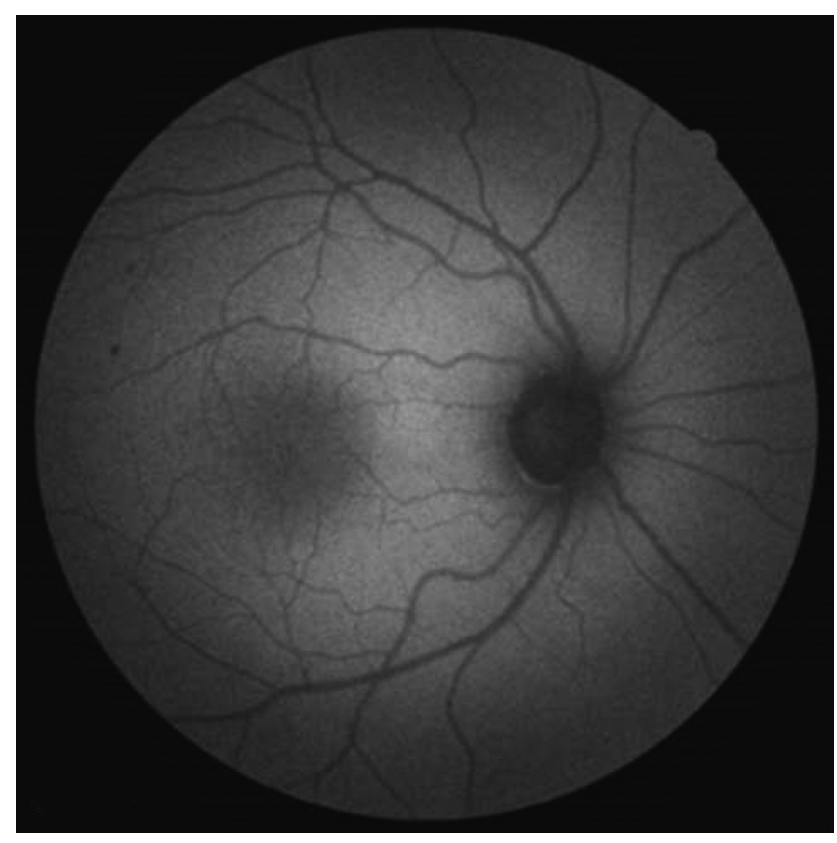

Figure $2 \mathrm{SW}-\mathrm{FAF}$ imaging in healthy subject. Abbreviation: SW-FAF, short-wavelength fundus autofluorescence. material (cells or liquid) located over the RPE and hyperautofluorescence signal, wherein the signal is abnormally bright. In this case, higher FAF intensity is due to an increase in lipofuscin accumulation, hyperautofluorescent structures, such as, intraretinal fluid, drusen in the subpigment epithelial space or older intraretinal and subretinal hemorrhages, and absence of signal blockage. ${ }^{14,15}$

\section{Age-related macular degeneration}

AMD is the most common cause of visual impairment and blindness in people older than 60 years. ${ }^{16}$ Even though the pathophysiology remains unknown, certain signs of oxidative damage are observed in its development, where lipofuscin accumulation plays an important role. ${ }^{17}$ AMD is classified into non-neovascular (dry or nonexudative) and neovascular (wet or exudative). The first visible change is the presence of drusen that is considered the clinical hallmark of nonneovascular AMD. This stage can progress to neovascular AMD or geography atrophy (GA), where areas of atrophy become confluent and cause visual loss. ${ }^{18}$

SW-FAF imaging shows numerous focal hyperautofluorescent spots, which may be due to the presence of lipofuscin, and hypoautofluorescent areas that correspond to RPE atrophy. ${ }^{19}$ These lesions are also observed by NIR-AF imaging, although hypautofluorescent spots have been detected with the same frequency than hyperautofluorescent spots. ${ }^{20}$

Drusen aspect depends on the size, composition, health of RPE and anterior retinal layers $;{ }^{17}$ thus, intermediate drusen shows a pattern of central hypoautofluorescence bordered by an annulus of hyperautofluorescence. This distribution corresponds to a central RPE atrophy surrounded by abnormal RPE. However, large and confluent drusen are seen as hyperautofluorescent lesions, ${ }^{21}$ and some FAF patterns in AMD may look normal as not all drusen can be easily spotted.

The presence of hyperautofluorescence annulus can be due to vertically disorganized RPE cells at the borders of atrophy ${ }^{22}$ or disorganized and vertically aligned RPE cells at the borders of atrophy. ${ }^{23}$ Also, the reduced FAF signal is due to atrophy of RPE leading to a loss of lipofuscin. ${ }^{7}$ Moreover, atrophy areas may be surrounded by hyperautofluorescent lesions that represent areas in the process of RPE cell dysfunction and progression of GA.

Neovascular AMD is characterized by the presence of choroidal neovascularization, and it is classified according to the location of neovascularization into type 1 (neovascularization is located under the RPE), type 2 (neovascularization is located above the RPE) and type 3 (neovascularization is located 
intraretinally). ${ }^{24} \mathrm{FAF}$ imaging is characterized by blockage of SW-AF and NIR-AF signals by choroidal neovascularization. ${ }^{20}$

In early stages of choroidal neovascularization, SW-AF intensity remains unaltered due to intact RPE and photoreceptor layers. Classic choroidal neovascularization (Figure 3:1A) appears hypofluorescent due to blockage of RPE caused by fibrovascular complex in the subretinal space (Figure $3: 1 \mathrm{~B}){ }^{25}$ Hemorrhages and exudates are observed as hypoautofluorescent signs due to excitation light absorption, which may become hyperautofluorescent in more advanced stages of the disease.

Kellner et al observed that the areas of exudation activity showed differences in FAF signal: in SW-FAF imaging, they were usually hyperautofluorescents, while in NIR-FAF imaging, these areas usually showed a decreased intensity. They suggested that this finding may explain why the area of increased intensity observed by SW-AF was larger than the area of increased NIR-AF signal in some cases of exudative AMD. ${ }^{20}$

Pilotto et al also evaluated the differences between both types of FAF imaging, and they correlated their findings with microperimetry data. They observed that the borders of GA atrophy showed abnormalities in NIR-FAF images, and they were strongly correlated with functionality shown in microperimetry than those observed in SW-FAF. They suggested that this observation corresponded to the areas of persistent photoreceptor layer over a damaged RPE, indicating that
NIR-FAF imaging may detect the areas of damaged RPE cells earlier than SW-FAF imaging. It could be because these areas increase melanogenesis process, melanoliposfuscin formation or oxidized melanin formation due to increased phagocytosis as a result of the photoreceptor degeneration. It is speculated that this increased NIR-AF signal, due to melanogenesis or phagocytosis, is followed by reduced NIRAF signal as a result of a decrease in melanosome number due to impairment of the photoreceptors. ${ }^{26}$

\section{DR and diabetic macular edema}

Diabetes mellitus (DM) is a metabolic syndrome with a high impact on health care due to its high mortality rate and increased prevalence, mainly in working-age population, with 320.5 million people being affected with DM. DR and diabetic macular edema (DME) are ocular complications of DM. The prevalence of any DR type is $34.6 \%$ among those with DM, and $12 \%$ of patients with DM develop DME. ${ }^{27}$

It is observed that the mechanism of lipofuscin accumulation in DR development is different from other retinal diseases, ${ }^{28}$ since in this disease, accumulation of lipofuscin is greater in microglia than in RPE. Thus, the pathophysiologic process of DR activates microglia, allowing the oxidation of proteins and lipids and, therefore, accumulation of lipofuscin granules in microglia. ${ }^{29}$

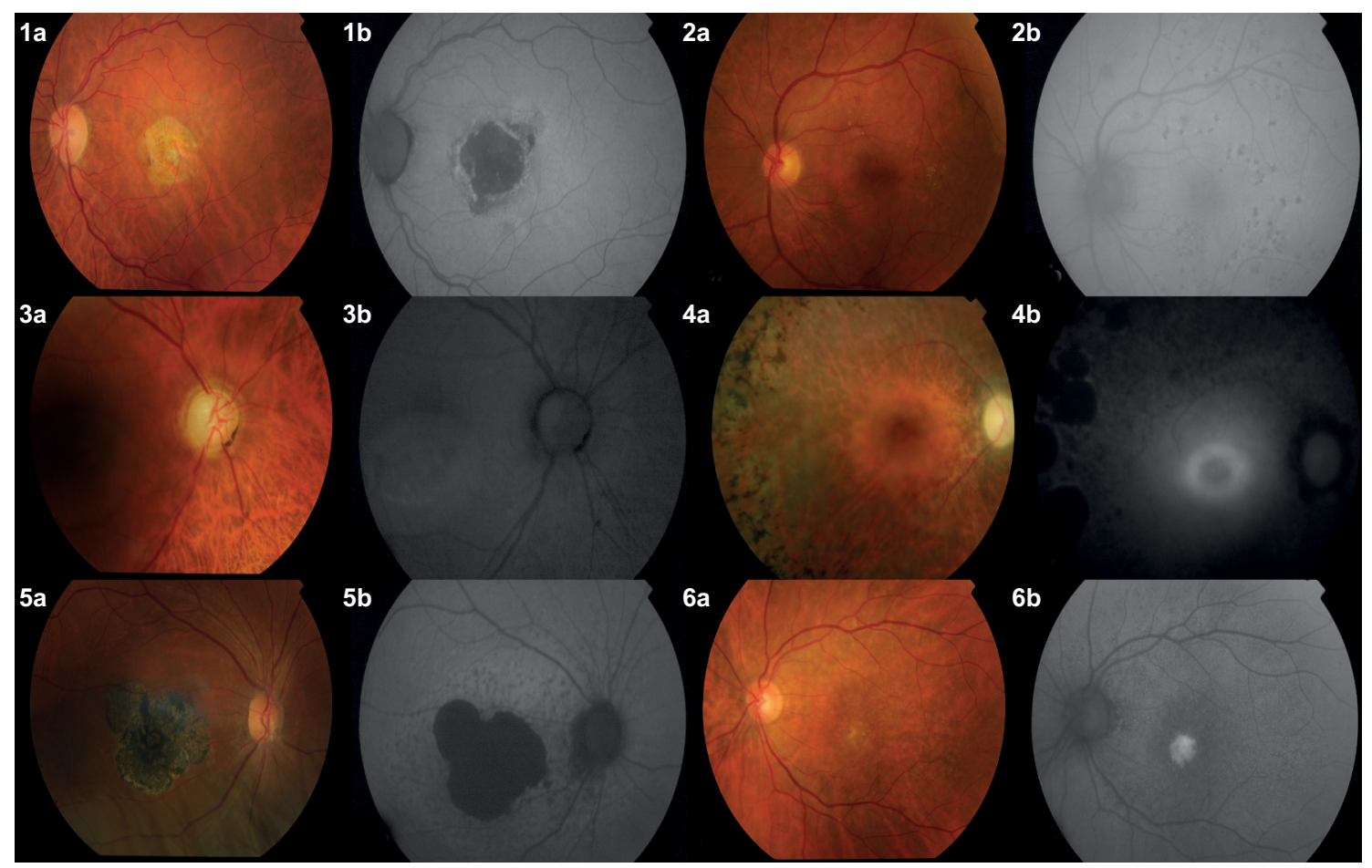

Figure 3 Color (a) and FAF (b) imaging of different retinal conditions: 1) Exudative age-related macular degeneration, 2) diabetic retinopathy, 3) central serous chorioretinopathy, 4) retinitis pigmentosa, 5) Stargardt disease, and 6) best vitelliform disease. All images have been provided by Clínica Universidad de Navarra. Abbreviation: FAF, fundus autofluorescence. 
Calvo-Maroto et al studied the usefulness of SW-FAF imaging to detect compatible signs with DR development in patients with DM using a non-mydriatic retinal camera. They observed that compatible signs with microaneurysms were observed as hypofluorescent granules and capillary dilation and hemorrhages as hypofluorescent areas (Figure 3:2B). They concluded that signs compatible with microaneurysms and capillary dilation were more evident in FAF and optimized-FAF images compared with color images; however, hard exudates were more difficult to identify in FAF and optimized-FAF images (Figure 3:2A and 2B). They also observed that optimized-FAF images show alterations compatible with DR not visible in color images, such as microaneurysms and capillary dilations. ${ }^{30}$

DME is one of the leading causes of severe visual loss in working-age population, and it can also be observed and assessed by FAF imaging. In clinical practice, DME assessment can be performed by fundus lamp biomicroscopy and optical coherence tomography (OCT), which is considered the morphologic gold standard for diagnosing DME. However, the presence of DME is associated with an increase in AF intensity that has been correlated with functional and structural impairment of the macula assessed by OCT. ${ }^{31}$

Patients with DME show SW-FAF patterns characterized by the presence of oval areas bordered by darker rims. FAF intensity depends on the location of cysts that may displace luteal pigment and avoid the normal blockage of foveal $\mathrm{FAF}^{32}$ The classification of DME based on SW-FAF patterns obtained by cSLO in patients with clinically significant macular edema includes four types: 1) normal FAF, 2) cystoid increased FAF, 3) spot increased FAF and 4) irregular decreased FAF. ${ }^{31}$

There are two possible hypotheses to explain these changes in SW-FAF imaging in patients with DME. In the first of these, it is hypothesized that an increase in FAF intensity observed in these patients is caused by accumulation of oxidative products induced by activated microglia. ${ }^{31}$ The second and the most accepted is the displacement of macular pigment. The FAF intensity of these ovoid lesions is similar to that of peripheral zones where the macular density is very low, and the intensity of the surrounding rims is the same as that of zones with high macular pigment concentration. ${ }^{33}$

When DME is improved, the FAF intensity does not show a significant reduction as the retinal cells and photoreceptors are capable of obtaining and stabilizing macular pigments. This persistent high FAF signal after DME resolution could indicate a decrease in macular cell function and/or persistent accumulation of oxidative products induced by microglial activation. ${ }^{34}$
DME assessment by SW-FAF imaging obtained by different devices, such as cSLO and digital non-mydriatic retinal cameras, shows certain differences in FAF intensity. Thus, Bessho et al observed an increased AF intensity by an excitation wavelength of $488 \mathrm{~nm}$ in eyes with cystoid macular edema, but only one eye showed increased AF by $580 \mathrm{~nm}$. They suggested that this difference could be due to the fact that increased AF by $488 \mathrm{~nm}$ is "pseudo" increased $\mathrm{AF}$ resulting from the reduction of blockage of AF by macular pigments. The lateral displacement of intraretinal cysts may cause a decrease in the density of macular pigments and alters the absorption of AF. This reduction of the macular pigment density is less influenced by $580 \mathrm{~nm}$, so this may result in low contrast of the cystoid lesions. ${ }^{35}$

Another study evaluated DME by a digital non-mydriatic retinal camera. Calvo-Maroto et al, in a blinded pilot study, observed that patients with DME showed an increase in FAF intensity in the macular area compared to those without DME. Moreover, FAF intensity that was measured in three macular zones (foveal, parafoveal and perifoveal zones) in those patients with DME showed higher values of FAF signal compared to those without DME (Calvo-Maroto et al, submitted for publication). The authors suggested that this hyperfluorescent zone located in the macular area may be due to the lateral displacement of the macular pigments or the presence of unknown fluorophore responsible for this fluorescence.

However, when DME is assessed by NIR-FAF imaging, a different pattern from that found in SW-FAF images can be observed. Yoshitake et al observed that patients with DME showed a decrease in AF signal in the macular area, a mosaic foveal pattern, hyper- and hypoautofluorescent granules and cystoid signal. They hypothesized that AF signal from RPE and choroides may be blocked by intraretinal lesions and subretinal fluids. However, hypofluorescent signs were not correlated with the findings observed in OCT, such as subretinal detachments and cysts. They suggested that DM may induce a decrease in melanin in RPE cells, allowing light and oxidative stress damage at the intercellular junctions of EPR and leading to an increase in RPE permeability. This hyperpermeability may contribute to migration of immune cells and an increase in macular thickness, causing the formation of a mosaic pattern. ${ }^{36}$

\section{Central serous chorioretinopathy}

CSC is characterized by serous retinal detachment or pigment epithelial detachment (Figure 3:3A). In most cases, CSC resolves spontaneously, although the visual acuity (VA) may 
not improve to the previous values of CSC episode due to photoreceptor and RPE damage. ${ }^{37}$

In the early stages of CSC, SW-FAF and NIR-FAF images show hypoautofluorescent areas correlated with serous retinal detachment due to blockage by subretinal fluid. These areas correspond to leakage areas when observed by fluorescein angiogram, ${ }^{38}$ where it also shows hypoautofluorescent signal. ${ }^{39}$ NIR-FAF imaging seems to be better to detect hypoautofluorescent leakage spots due to bright luminescence in the macular area compared to dark luminescence in SW-FAF imaging. ${ }^{40}$

During CSC progression, both types of FAF images show granular hyperautofluorescence ${ }^{38}$ (Figure 3:3B) that corresponds to subretinal precipitates on OCT assessment. ${ }^{39}$

Several authors have investigated possible correlations between FAF signal and VA after resolution of subretinal fluid. Oh et al observed that gray values at 350 and $1200 \mu \mathrm{m}$ circles around the fovea were correlated with best-corrected VA and microperimetry data in SW-FAF imaging; however, this correlation was not found in NIR-FAF imaging, ${ }^{41}$ although Sekiryu et al demonstrated that changes in NIRFAF imaging during follow-up period were correlated with the final best-corrected VA. ${ }^{42}$

\section{Retinitis pigmentosa}

$\mathrm{RP}$ represents a group of inherited retinal diseases characterized by photoreceptor degeneration (Figure 3:4A), which can lead to blindness in the advanced stages of disease. ${ }^{43}$

In certain patients with RP, SW-FAF imaging shows a FAF pattern where an annular ring of hyperautofluorescence can be observed which corresponds to the border or inner/outer segment junction disruption RP (Figure 3:4B) ${ }^{43}$ This pattern can also be observed in NIR-FAF imaging. ${ }^{44}$ However, differences have been observed between both types of FAF imaging, such as inner border of ring on NIR-FAF imaging which is closer to fovea than on SW-FAF. It has been observed that this inner border coincides with a location where the hyperreflective inner segment ellipsoid band on spectral domain-OCT seems to be partially preserved, even intact. Duncker et al suggested that NIR-FAF imaging could detect a process in RP progression before fluorophore formation responsible for the SW-FAF signal. ${ }^{44}$

Electroretinography is considered the gold standard for diagnosis and monitoring of RP progression and shows spatial preservation of visual sensitivity within the ring on SW-FAF and NIR-FAF imaging, which is decreased on the ring and also outside the outer border of the ring. ${ }^{45,46}$

\section{Macular dystrophies}

\section{Stargardt disease}

Stargardt disease is the most common hereditary juvenile macular dystrophy. The autosomal recessive mutation in the $A B C A 4$ gene causes a progressive loss of central vision that typically occurs in the first two decades of life. An abnormal outer segment degradation, lipofuscin accumulation and central degeneration of the RPE and photoreceptor layer are consequences of this mutation. ${ }^{47}$

These patients show an ophthalmoscopic pattern characterized by central macular atrophy (Figure 3:5A) and yellowwhite flecks at the posterior pole, mainly at the RPE level. ${ }^{47}$

Duncker et al studied the differences between SW-FAF and NIR-FAF imaging, and for this reason, they classified patients based on different patterns observed in both types of images into 1) central lesion with jagged border (Figure 3:5B), 2) lesion with extensive fundus changes, 3) central lesion with smooth border and hyperautofluorescent SW-AF and NIR-AF ring, 4) central lesion with smooth border and no hyperautofluorescent NIR-AF ring and 5) discrete central lesions better visualized in NIR-AF images. ${ }^{48}$

Although there were similarities between SW-FAF and NIR-FAF patterns, they also observed several differences, such as the area of central ellipsoid that corresponds to zone of photorreceptor loss. When NIR-AF was used, the signal was low, while with SW-AF, the signal was increased, decreased or similar to surrounding areas. Also, the zone of signal reduced NIR-AF was larger than the area. ${ }^{48}$

Another difference between both types of images is the appearance of flecks. With disease progression, flecks emerge along the central to the peripheral axis of the retina. While in SW-FAF images flecks are usually hyperautofluorescent, in NIR-FAF images flecks are most often hypoautofluorescent. However, the SW-AF signal of flecks undergoes decay with time. ${ }^{49}$

\section{Best vitelliform disease}

Best vitelliform macular disease (BVMD) is a rare disorder inherited in an autosomal dominant mutation in BEST1 gene that transcribes the bestropin-1 protein located on the membrane of RPE cells. ${ }^{50}$

All ophthalmoscopic features of BVMD are restricted to the macula (Figure 3:6A); thus, a quantitative FAF study revealed that areas located outside the central lesion do not exhibit increased lipofuscin concentration. ${ }^{50}$

Querques et al studied SW-FAF imaging in five stages of BVMD progression: previtelliform, vitelliform, pseudohypopyon, vitelliruptive and atrophic. Previtelliform stage showed 
minimal hyperautofluorescence intensity (Figure 3:6B). Vitelliform stage was characterized by marked hyperautofluorescence in the macula. The pseudohypopyon stage showed a gravitational layer of hyperautofluorescence located under isoautofluorescent fluid. The vitelliruptive stage showed a dark lesion bordered by hyperautofluorescent material. The atrophic stage was characterized by a decreased signal due to chorioretinal atrophy. ${ }^{51}$

Parodi et al observed six different phenotypes of this disease with SW-FAF and NIR-FAF images, which were classified into normal AF, hyperautofluorescent, hypoautofluorescent, patchy, multifocal and spoke-like patterns. The patchy subtype was most frequent, followed by the hyperautofluorescent and hypoautofluorescent patterns. These patterns did not correlate with the stages of progression of this disease. ${ }^{52}$

In conclusion, FAF imaging pattern depends on the excitation wavelength used due to excitation of different fluorophores, while in SW-FAF imaging, the fluorophores, while in SW-FAF imaging the fluorophore excited is lipofuscin, in NIR-FAF imaging it is melanin and/or compounds related to melanin, and therefore, both types of images show differences in distribution and intensity of FAF signal. These differences can be observed at different stages of several diseases, providing information about the pathogenesis and progression of retinal diseases. Thus, FAF images may be considered as a useful tool for the optometrist and medical practitioners for the early detection, assessment and monitoring of retinal disease. Considerable research, however, still needs to be carried out to explore the full potential of the technique, develop metrics and analysis methods for FAF images, as well as to know the full range of applicability for detecting abnormality in ocular tissues from $\mathrm{AF}$, not only within the retina but also in the anterior segment.

\section{Disclosure}

The authors report no conflicts of interest in this work.

\section{References}

1. Boulton M, Rózanowska M, Rózanowski B. Retinal photodamage. J Photochem Photobiol B Bio. 2001;64(2-3):144-161.

2. Rovati L, Docchio F. Autofluorescence methods in ophthalmology. J Biomed Opt. 2004;9(1):9-21.

3. Delori FC, Dorey CK, Staurenghi G, Arend O, Goger DG, Weiter JJ. In vivo fluorescence of the ocular fundus exhibits retinal pigment epithelium lipofuscin characteristics. Invest Ophthalmol Vis Sci. 1995;36(3):718-729.

4. Shen Y, Xu X, Liu K. Fundus autofluorescence characteristics in patients with diabetic macular edema. China Me J (Engl). 2014;127(8):1423-1428.

5. Durrani K, Foster CS. Fundus autofluorescence imaging in posterior uveitis. Semin Ophthalmol. 2012;27(5-6):228-235.

6. Sparrow JR, Fishkin N, Zhou J, et al. A2E, a byproduct of the visual cycle. Vision Res. 2003;23(28):2983-2990.
7. Sepah YJ, Akhatar A, Sadiq MA, et al. Fundus autofluorescence imaging: fundamentals and clinical relevance. Saudi J Ophthalmol. 2014;28(2):111-116.

8. Schutt F, Bergmann M, Holz FG, Kopitz J. Proteins modified by malondialdehyde, 4-hydroxynonenal, or advanced glycation end products in lipofuscin of human retinal pigment epithelium. Invest Ophthalmol Vis Sci. 2003;44(8):3663-3668.

9. Calvo-Maroto AM, Esteve-Taboada JJ, Domínguez-Vicent A, PérezCambrodí RJ, Cerviño A. Confocal scanning laser ophthalmoscopy versus modified conventional fundus camera for fundus autofluorescence. Expert Rev Med Devices. 2016;13(10):965-978.

10. Vujosevic S, Casciano M, Pilotto E, Boccassini B, Varano M, Midena E. Diabetic macular edema: fundus autofluorescence and functional correlations. Invest Opthalmol Vis Sci. 2011;52(1):442-448.

11. Keilhauer CN, Delori FC. Near-infrared autofluorescence imaging of the fundus: visualization of ocular melanin. Invest Ophthalmol Vis Sci. 2006;47(4):3556-3564.

12. Feeney-Burns L, Hilderbrand ES, Eldridge S. Aging human RPE: morphometric analysis of macular, equatorial, and peripheral cells. Invest Ophthalmol Vis Sci. 1984;25(2):195-200.

13. Schimtz-Valckenberg, Holz FG, Bird AC, Apide RF. Fundus autofluorescence imaging: review and perspectives. Retina. 2008(3);28(3):385-409.

14. Samy A, Lightman S, Ismetova F, Talat L, Tomkins-Netzer O. Role of autofluorescence in inflammatory/infective diseases of the retinal and choroid. J Ophthalmol. 2014;2014;418193.

15. Spaide RF. Autofluorescence imaging with a fundus camera. In: Holz FSVS, Spaide RF, Bird AC, editors. Atlas of Fundus Autofluorescence Imaging. New York, NY: Springer-Verlag; 2007:207-239.

16. Wong WL, Su X, Li X, et al. Global prevalence of age-related macular degeneration and disease burden projection for 2020 and 2040: a systemic review and meta-analysis. Lancet Glob Health. 2014:2(2): e106-e116.

17. Lois N, Owens SL, Coco R, Hopkins J, Fitzke FW, Bird AC. Fundus autofluorescence in patients with age-related macular degeneration and high risk of visual loss. Am J Ophtalmol. 2002;133(3):341-349.

18. Bird AC, Bressler NM, Bressler SB, et al. An international classification and grading system for age-related maculapathy and age-related macular degeneration: the International ARM Epidemiological Study Group. Surv Ophthalmol. 1995;39(5):367-374.

19. Spaide RF. Fundus autofluorescence and age-related macular degeneration. Ophthalmology. 2003;110(2):392-399.

20. Kellner U, Kellner S, Weinitiz S. Fundus autofluorescence (488 NM) and near-infrared autofluorescence $(787 \mathrm{NM})$ visualize different retinal pigment epithelium alterations in patients with age-related macular degeneration. Retina. 2010;30(1):6-15.

21. Delori FC, Fleckner MR, Goger DG, Weiter JJ, Dorey CK. Autofluorescence distribution associated with drusen in age-related macular degeneration. Invest Ophthalmol Vis Sci. 20014;41(2):496-504.

22. Ablonczy Z, Higbee D, Anderson DM, et al. Lack of correlation between the spatial distribution of $\mathrm{A} 2 \mathrm{E}$ and lipofuscin fluorescence in the human retinal pigment epithelium. Invest Ophthalmol Vis Sci. 2013;54(8):5535-5542.

23. Rudolf M, Vogt SD, Curcio CA, et al. Histologic basis of variations in retinal pigment epithelium autofluorescence in eyes with geographic atrophy. Ophthalmology. 2013;120(4):821-828.

24. Nagiel A, Freund KB, Jung JJ, et al. Origin and behavior of type 3 neovascularization revealed by spectral-domain optical coherence tomography. Invest Ophthalmol Vis Sci. 2014;55(13):3938.

25. Camacho N, Barteselli G, Nezgoda JT, et al. Significance of the hyperautofluorescent ring associated with choroidal neovascularization in eyes undergoing anti-VEGF therapy for wet age-related macular degeneration. Br J Ophthalmol. 2015;99(9):1277-1283.

26. Pilotto E, Vujosevic S, Melis R, et al. Short wavelength fundus autofluorescence versus near-infrared fundus autofluorescence, with microperimetric correspondence, in patients with geographic atrophy due to age-related macular degeneration. Br J Ophthalmol. 2011;95(8): $1140-1144$. 
27. Gundogan FC, Yolcu U, Akay F, et al. Diabetic macular edema. PakJ Med Sci. 2016;32(2):505-510.

28. Spaide R. Autofluorescence from the outer retinal and subretinal space: hypothesis and review. Retina. 2008;28(1):5-35.

29. Xu H, Chen M, Maninvannan A, Lois N, Forrester JV. Age-dependent accumulation of lipofuscin in perivascular and subretinal microglia in experimental mice. Aging Cell. 2008;7(1):58-68.

30. Calvo-Maroto AM, Esteve-Taboada JJ, Perez-Cambrodí RJ, MadridCosta D, Cerviño A. Pilot study of visual function and fundus autofluorescence assessement in diabetic patients. $J$ Ophthalmol. 2016;2016:1287847.

31. Vujosevic S, Casciano M, Pilotto E, Boccassini B, Varano M, Midena E. Diabetic macular edema: fundus autofluorescence and functional correlations. Invest Ophthalmol Vis Sci. 2011;52(1):442-448.

32. McBain VA, Forrester JV, Lois N. Fundus autofluorescence in the diagnosis of cystoid macular oedema. Br J Ophthalmol. 2008;92(7):946-949.

33. Waldstein SM, Hickey D, Mahmud I, Kiire CA, Charbel Issa P, Chong NV. Two-wavelength fundus autofluorescence and macular pigment optical density imaging in diabetic macular oedema. Eye (Lond). 2012;26(8):1078-1085.

34. Chung H, Park B, Shin HJ, Kim HC. Correlation of fundus autofluorescence eith spectral-domain optical coherence tomography and vision in diabetic macular edema. Ophthalmology. 2012:119(5):1056-1065.

35. Bessho K, Gomi F, Harino S, et al. Macular autofluorescence in eyes with cystoid macular edema, detected with $488 \mathrm{~nm}$-excitation but not with 580nm-excitation. Graefes Arch Clin Exp Ophthalmol. 2009;247(6):729-734.

36. Yoshitake S, Murakami T, Horii T, et al. Qualitative and quantitative characteristics of near-infrared autofluorescence in diabetic macular edema. Ophthalmology. 2014;121(5):1036-1044.

37. Abouammoh MA. Advances in the treatment of central serous chorioretinopathy. Saudi J Ophthalmol. 2015;29(4):278-286.

38. Ayata A, Tatlipinar S, Kar T, Unal M, Ersanli D, Bilge AH. Near-infrared and short-wavelength autofluorescence imaging in central serous chorioretinopathy. Br J Ophthalmol. 2009;93(1):79-82.

39. Iacono P, Battaglia PM, Papayannis A, et al. Acute central serous chorioretinopathy: a correlation study between fundus autofluorescence and spectral-domain OCT. Graefes Arch Clin Exp Ophthalmol. 2015;253(11):1889-1897.

40. Skondra D, Papakostas TD, Hunter R, Vavvas DG. Near infrared autofluorescence imaging of retinal diseases. Semin Ophthalmol. 2012:27(5-6):202-208.
41. Oh J, Kim SW, Kwon SS, Oh IK, Huh K. Correlation of fundus autofluorescence gray values with vision and microperimetry in resolved central serous chorioretinopathy. Invest Ophthalmol Vis Sci. 2012;53(1):179-184.

42. Sekiryu T, Iida T, Maruko I, Saito K, Kondo T. Infrared fundus autofluorescence and central serous chorioretinopathy. Invest Ophthalmol Vis Sci. 2010;51(10):4956-4962.

43. Lee TJ, Hwang JC, Chen RW, et al. The role of fundus autofluorescence in late-onset retinitis pigmentosa (LORP) diagnosis. Ophthalmic Genet. 2014;35(3):170-179.

44. Duncker T, Tabacaru MR, Lee W, Tsang SH, Sparrow JR, Greenstein VC. Comparison of near-infrared and short-wavelength autofluorescence in retinitis pigmentosa. Invest Ophthalmol Vis Sci. 2013;54(1):585-591.

45. Robson RB, Saihan Z, Jenkins SA, et al. Funcional characterization and serial imaging of abnormal fundus autofluorescence in patients with retinitis pigmentosa and normal visual acuity. Br J Ophthalmol. 2006;90(4):472-479.

46. Greenstein VC, Duncker T, Holopigian K, et al. Structural and functional changes associated with normal and abnormal fundus autofluorescence in patients with retinitis pigmentosa. Retina. 2012;32(2):349-357.

47. Fujinami K, Lois N, Davidson AE. A longitudinal study of Stargardt disease: clinical and electrophysiologic assessement, progression, and genotype correlations. Am J Ophthalmol. 2013;155(6):1075-1888.

48. Duncker T, Marsiglia M, Lee W, et al. Correlations among near-infrared and short-wavelength autofluorescence and spectral-domain optical coherence tomography in recessive Stargardt disease. Invest Ophthalmol Vis Sci. 2014;55(12):8134-8143.

49. Sparrow JR, Marsiglia M, Allikmets R, et al. Flecks in recessive Stargardt disease: short-wavelength autofluorescence, near-infrared autofluorescence, and optical coherence tomography. Invest Ophthalmol Vis Sci. 2015;56(8):5029-5039.

50. Duncker T, Greenberg JP, Ramachandran R, et al. Quantitative fundus autofluorescence and optical coherence tomography in best vitelliform macular dystrophy. Invest Ophthalmol Vis Sci. 2014;55(3):1471-1482.

51. Querques G, Zerbib J, Georges A, et al. Multimodal analysis of the progression of best vitelliform macular dystrophy. Mol Vis. 2014;20:575-592.

52. Parodi MB, Iacono P, Campa C, Del Turco C, Bandello F. Fundus autofluorescence patterns in best vitelliform macular dystrophy. Am J Ophthalmol. 2014;158(5):1086-1092.
Clinical Optometry

\section{Publish your work in this journal}

Clinical Optometry is an international, peer-reviewed, open access journal publishing original research, basic science, clinical and epidemiological studies, reviews and evaluations on clinical optometry. All aspects of patient care are addressed within the journal as well as the practice of optometry including economic and business analyses. Basic and clinical Submit your manuscript here: https://www.dovepress.com/clinical-optometry-journal

\section{Dovepress}

research papers are published that cover all aspects of optics, refraction and its application to the theory and practice of optometry. The manuscrip management system is completely online and includes a very quick and fair peer-review system, which is all easy to use. Visit http://www.dovepress. com/testimonials.php to read real quotes from published authors. 